\title{
Cognição e eletrofisiologia: uma revisão crítica das perspectivas nacionais
}

\author{
Antonio Jaeger' - Washington University, Saint Lowis, Estados Unidos \\ Maria Alice de Mattos Pimenta Parente - Universidade Federal do Rio Grande do Sul, Porto Alegre, Brasil
}

\begin{abstract}
Resumo
A técnica eletrofisiológica Potenciais Relacionados ao Evento (PRE) vem sendo amplamente utilizada para o estudo da atividade cerebral correspondente a processos cognitivos específicos. O presente artigo apresenta uma revisão crítica dos estudos empregando PRE desenvolvidos em universidades brasileiras, enfatizando especialmente seus objetivos principais e abordagens metodológicas. Os estudos examinados investigaram efeitos eletrofisiológicos tradicionalmente reportados na literatura em PRE, como os efeitos denominados P300, N400 e MMN, proporcionando resultados relevantes teoricamente, bem como demonstrando a utilidade do PRE para a investigação de diversos temas de pesquisa. Possibilidades referentes a abordagens metodológicas alternativas para a análise dos PREs, assim como o emprego desta técnica no estudo de diversos processos psicológicos, são examinados e discutidos.

Palavras-chave: Eletrofisiologia, Cognição, Neurociência.
\end{abstract}

Cognition and electrophysiology: a critical review on the national perspectives

\begin{abstract}
The electrophysiological technique Event-related potentials (ERP) has been widely employed in the study of brain activity reflecting specific cognitive processes. This current article presents a critical review of the ERP studies conducted in Brazilian universities, with special emphasis on their main goals and methodological approaches. The examined studies investigated electrophysiological effects traditionally reported in the ERP literature, as the P300, N400 and MMN effects, providing theoretically relevant results and demonstrating the usefulness of this technique for the investigation of several research topics. Possibilities concerning alternative methodological approaches on ERP analysis and the employment of this technique in the study of a variety of psychological processes are examined and discussed.

Keywords: Electrophysiology, Cognition, Neuroscience.
\end{abstract}

A utilização de técnicas não-invasivas que
extraem representações mensuráveis do funcionamento cerebral humano ante manipulações experimentais específicas é uma das mais promissoras abordagens atualmente empregadas no estudo da cognição (Nunez \& Srinivasan, 2006). A técnica denominada "potenciais relacionados ao evento" (PRE), também conhecida como "potencial evocado", consiste em um procedimento pelo qual se extrai, por meio de eletrodos distribuídos sobre o escalpo, potenciais elétricos gerados no cérebro, os quais são originalmente sinais de eletroencefalograma (EEG), relacionando-os diretamente a manipulações experimentais específicas. O sinal de EEG extraído, nesse caso, é relacionado a intervalos determinados de tempo, os quais são diretamente relacionados à apresentação dos estímulos experimentais. O objetivo principal subjacente a esse procedimento é relacionar as ativações eletrofisiológicas geradas pelo cérebro a processos cognitivos específicos desempenhados ante determinadas tarefas experimentais (Handy, 2004; Lopes da Silva, 2004).

Atualmente, existe uma série de técnicas que proporcionam monitoramento da atividade cerebral humana durante o desempenho de tarefas

${ }^{1}$ E-mail: ajaeger@artsci.wustl.edu e antonio.jaeger@gmail.com experimentais, como, por exemplo, a ressonância magnética funcional (RMF) (Huettel, Song \& McCarthy, 2004) e a tomografia por emissão de pósitrons (TEP) (Bailey, Townsend, Valk \& Maisey, 2005). Porém, essas técnicas proporcionam informações quanto à atividade cerebral baseando-se no aumento da circulação sanguínea iniciada logo após que determinada região do cérebro é ativada (Turner, Howseman, Rees, Josephs \& Friston, 1998). Isto é, são técnicas que monitoram de maneira indireta a atividade cerebral subjacente aos processos cognitivos. A extração de PREs, por consistirem em sinais elétricos resultantes da atividade sináptica simultânea de conjuntos de neurônios, é uma técnica que extrai sinais que representam de maneira direta a atividade cerebral humana (Bressler, 2002).

Consequentemente, uma das principais vantagens do PRE em relação a outras técnicas é sua impecável resolução temporal, isto é, enquanto técnicas como a RMF proporcionam imagens da atividade cerebral em intervalos de 2 a 3 segundos, os PREs são extraídos em tempo real, proporcionando a análise das ativações elétrico-cerebrais na ordem de unidades de milissegundos (Rugg, 1997). Sendo assim, embora a RMF possua uma grande precisão espacial, a resolução temporal com que os sinais de PRE podem ser extraídos faz deste a ferramenta de escolha para a 
investigação de aspectos temporais de diversos processos cognitivos (Handy, 2004).

Essa técnica também apresenta uma importante vantagem econômica em relação a outras técnicas não-invasivas de monitoração da atividade cerebral humana, como a RMF ou a TEP (Niedermeyer \& Lopes da Silva, 2004). Isto é, possui baixo custo de instalação e baixo custo de utilização, permitindo uma implementação mais ampla da mesma em universidades de países em desenvolvimento, como o Brasil.

Tendo-se em vista as vantagens técnicas e metodológicas desse procedimento, assim como sua viabilidade econômica, o objetivo principal deste estudo consistiu em descrever e discutir criticamente os objetivos, a metodologia e os principais achados de pesquisas empíricas originais que utilizaram extração de PREs e foram desenvolvidas dentro de sistemas de pós-graduação de universidades brasileiras. Procura-se ainda, com este estudo, colocar a pesquisa nacional em PRE em perspectiva quanto ao contexto científico global, contrastando-se as abordagens metodológicas da mesma com as abordagens metodológicas empregadas em estudos de alto impacto na literatura científica internacional. Dessa maneira, busca-se proporcionar uma visão mais abrangente das possíveis limitações técnicas inerentes às abordagens metodológicas nacionais atuais, assim como possíveis áreas de expansão e possibilidades futuras para a pesquisa em PRE no Brasil.

Para alcançar esse objetivo, os autores realizaram um levantamento dos estudos com essa metodologia publicados em periódicos indexados às base de dados Scielo, Pubmed e PsycInfo, com datas de publicação entre janeiro de 2000 e agosto de 2008 . Foram incluídos na busca bibliográfica estudos em PRE desenvolvidos em universidades nacionais ou estudos em cooperação com universidades estrangeiras em que a coleta de dados tenha sido desenvolvida em universidades nacionais e os principais autores sejam vinculados a essas universidades. Excluiu-se da busca bibliográfica estudos que não possuem como objetivo investigar PREs em relação a processos cognitivos, ou seja, estudos que buscaram investigar ativações eletrofisiológicas eliciadas nos aparelhos sensoriais, assim como estudos que investigaram potenciais oscilatórios de EEG ou EEG quantitativo não foram incluídos no presente estudo.

A metodologia de pesquisa em PREs implica, de modo geral, buscar, nos dados, fenômenos eletrofisiológicos específicos eliciados por manipulações experimentais. Um exemplo disso é a detecção de picos ou depressões na onda elétrica gerada no escalpo que se diferenciam de acordo com a condição experimental, passando essas características passam a compôr o que é chamado de "componente" de interesse. Com esta abordagem, estas características podem ser encontradas analisando-se aspectos simples dos dados, como a amplitude (microvolts) ou a latência (milissegundos) da onda (Handy, 2004; Lopes da Silva, 2004).

Em virtude do procedimento PRE estar sendo empregado em pesquisa há várias décadas (Sutton, Braren, Zubin \& John, 1965), certa quantidade de componentes já foi submetida a inúmeros testes, o que proporciona um alto grau de confiabilidade aos mesmos (Kutas \& Dale, 1997). Um exemplo importante do fato é o componente denominado P300 ou P3, onde a letra $\mathrm{P}$ significa que é um componente que possui voltagem positiva e o número 300 indica que o mesmo inicia 300 milissegundos após a apresentação do estímulo (Polich, 2007). Esse componente é eliciado principalmente por tarefas denominadas oddball, nas quais os sujeitos são instruídos a detectar estímulos-alvo pouco frequentes, que ocorrem imprevisivelmente em uma sequência de estímulos não-alvo e altamente frequentes (Kutas \& Dale, 1997). Esse efeito é eliciado independentemente da modalidade sensorial e postulado como um representante de diferentes processos cognitivos, como por exemplo, a atualização da memória de trabalho (Donchin \& Coles, 1988) e a transferência de informações para a consciência (Picton, 1992).

Outros exemplos de componentes importantes são o Mismatch Negativity (MMN, também denominado N200) e o N400. O primeiro efeito é normalmente eliciado pelo paradigma oddball mais rapidamente do que o P300 (aproximadamente 200 milissegundos após apresentação do estímulo) e, de acordo com estudos sobre atenção, está associado ao processamento cortical automático das informações físicas disponíveis nos aparelhos sensoriais. O segundo, é um dos componentes mais estudados em linguagem, definido como um correlato eletrofisiológico do processamento de sentenças anômalas versus sentenças corretamente construídas (Coles \& Rugg, 1995).

Em contrapartida ao emprego desses paradigmas tradicionalmente utilizados, estudos que visam abordar modelos teóricos atuais no âmbito das ciências cognitivas empregam o PRE juntamente com protocolos experimentais que proporcionem a investigação de diversos processos cognitivos, como, por exemplo, percepção, memória e tomada de decisões. Dessa forma, questões teóricas importantes das ciências cognitivas que atualmente contam com o suporte de dados de PRE para sua investigação são, por exemplo, a busca por uma compreensão das 
dissociações entre memórias implícitas e explícitas (Badgaiyan \& Posner, 1997), e a resolução do debate entre dois modelos teóricos distintos, denominados "Modelo de processamento duplo" e "Detecção de sinal", que procuram descrever a natureza do processo de memória de reconhecimento (Rugg \& Curran, 2007).

No Brasil, a maioria dos estudos se baseia em paradigmas mais tradicionais, utilizando o oddball e buscando eliciar o componente P300 (Sutton \& colaboradores, 1965). Dentre os 22 estudos brasileiros com extração de PREs encontrados na literatura disponibilizada pelas bases de dados online acima citadas, entre o período de 2000 e 2008, 18 visavam eliciar esse componente. Um estudo investigou o MMN e três investigaram o N400. Para a extração do MMN, o paradigma oddball foi utilizado, e para o $\mathrm{N} 400$, os pesquisadores utilizaram o paradigma verbcomplement merge, ou a apresentação de palavras e pseudopalavras. Assim, com o intuito de facilitar a compreensão do presente texto, os trabalhos citados serão distribuídos conforme o componente abordado e conforme as variáveis independentes investigadas por cada estudo.

\section{Revisão bibliográfica}

\section{Componente P300}

Como mencionado anteriormente, O componente P300 é eliciado pelo paradigma oddball e foi reportado em publicação científica pela primeira vez em 1965 (Sutton \& colaboradores, 1965). Desde então, inúmeros experimentos têm utilizado esse paradigma experimental em pesquisa (Polich, 2007), sendo que uma quantidade significativa de estudos tem relacionado-o com outras variáveis, como, por exemplo, transtornos psiquiátricos (transtorno de humor bipolar e esquizofrenia) (Thaker, 2008), e seus substratos neuroquímicos, como os sistemas dopaminérgicos e serotoninérgicos (Frodl-Bauch, Bottlender \& Hegerl, 1999; Hegerl, 2004). Os estudos que visam eliciar o P300, normalmente utilizam uma quantidade pequena de eletrodos distribuídos sobre o escalpo para captar o sinal de PRE (entre 2 e 4), tradicionalmente colocados sobre a linha central do escalpo (midline). As principais características dos dados a serem observadas são a amplitude do efeito (microvolts) e sua latência (milissegundos).

Foram encontrados três estudos nacionais sobre o P300 que possuíam um caráter normativo (Veiga \& colaboradores, 2004; Colafêmina, Fellipe, Junqueira \& Frizzo, 2000; Franco, 2001), ou seja, buscavam estabelecer, em termos de latência e amplitude, o efeito P300 padrão para sujeitos adulto- jovens saudáveis. Todos os três estudos reportaram alta variabilidade nos valores da latência e amplitude, o que corrobora dados da literatura internacional (Polich, 2007) e torna pouco prática a utilização dessa técnica para fins clínicos e diagnósticos.

No que se refere à influência do envelhecimento sobre o componente P300, encontrouse apenas um estudo (Matas, Santos Filha, Okada \& Resque, 2006), o qual avaliou o P300 em três grupos de idade de indivíduos acima de 50 anos (50 a 59, 60 à 69 e 70 a 79 anos) e demonstrou diferenças significativas entre eles. Os autores demonstram que a latência desse efeito aumentou conforme aumentava a idade dos sujeitos. Esta correlação entre aumento de latência e aumento de idade foi sugerida pelos autores como sendo resultado de mudanças cerebrais estruturais relacionadas ao envelhecimento.

Foram encontrados cinco estudos nacionais investigando a influência da ingestão de substâncias sobre este componente. Um desses estudos investigou a influência da cafeína e do bromazepam no efeito P300 (Montenegro \& colaboradores, 2005), demonstrando que as duas substâncias possuem efeitos modulatórios distintos sobre esse componente. Quando se investigou somente a influência da cafeína (Deslandes \& colaboradores, 2005), efeitos modulatórios também estavam presentes, mas quando a influência do bromazepam foi estudada separadamente, não houve qualquer modulação dessa substância sobre o P300 (Puga \& colaboradores, 2005). Um estudo duplo cego investigou a influência do lorazepam e do flunitrazepam sobre esse componente, demonstrando importantes diferenças na latência relacionadas à ingestão de cada uma das substâncias (Pompéia \& colaboradores, 2000). Ainda no que se refere à influência de substâncias no P300, um estudo demonstrou que crianças com transtorno de déficit de atenção e hiperatividade, às quais medicações específicas para esse diagnóstico estavam sendo administradas, não apresentaram diferenças ao eliciar esse componente em relação a crianças com o mesmo diagnóstico, às quais essas medicações não estavam sendo administradas (Shochat, Scheuer \& Andrade, 2002).

Alguns trabalhos investigaram diferenças no P300 relacionadas a diagnósticos clínicos. Um desses estudos incluiu em sua amostra indivíduos com a condição clínica diabetes mellitus e indivíduos saudáveis (Alvarenga \& colaboradores, 2005), demonstrando diferenças significativas entre esses grupos no que se refere à latência do componente em questão. Isto é, o efeito P300 dos indivíduos com diabetes mellitus ocorreu com atraso em relação ao efeito P300 dos indivíduos saudáveis. Alterações semelhantes na latência do P300 
também foram demonstradas em sujeitos portadores do vírus da AIDS (Matas, Juan \& Nakano, 2006), assim como para sujeitos com diferentes graus de perda auditiva (Reis \& Iório, 2007).

Um estudo desenvolvido por VianaWackermann, Furtado, Esser, Schmidt e Laucht (2007) demonstrou uma importante diminuição da amplitude desse componente quando os participantes (crianças) possuíam pais com história de alcoolismo ou delinquência. Outro estudo que investigou o P300 em crianças avaliou esse efeito de acordo com diferentes rendimentos escolares (Aquino, Bardão, Colafêmina, Gonçalvez \& Casagrande-Souza, 2000), demonstrando relações entre desempenho escolar e a latência do P300, e discutindo a utilidade desse componente para a identificação de problemas de aprendizado escolar. Quando se investigou a presença ou ausência de epilepsia, relacionando-a a rendimento escolar, resultados significativos foram obtidos somente quanto a rendimento escolar, demonstrando que a latência do P300 de crianças com bom rendimento escolar era significativamente menor em comparação com crianças com mau desempenho escolar, com eplepsia ou não (Visioli-Mello \& Rotta, 2000).

Estudos com extração de P300 que tiveram como objetivo investigar questões metodológicas envolvidas na utilização desse componente, para fins clínicos ou de pesquisa, também foram desenvolvidos. Um deles investigou diferenças na avaliação de examinadores no que se refere a esse efeito. Isto é, os autores apontaram para a diversidade metodológica envolvida na identificação do P300 e para as diferentes interpretações que diferentes examinadores podem atribuir aos termos empregados na literatura referente ao P300 (Junqueira \& Colafêmina, 2002). Os autores concluem que, para tornar a utilização e interpretação do P300 uniforme entre diferentes examinadores e laboratórios, os pesquisadores devem desenvolver experimentos em que a análise do P300, assim como sua interpretação, sejam realizadas de acordo com critérios pre determinados. Outro estudo investigou a aplicabilidade da apresentação de estímulos auditivos em campo livre em contraste com a apresentação em fones (Duarte, Alvarenga \& Costa, 2004), demonstrando que o P300 pode ser igualmente extraído em ambas as situações experimentais.

Por fim, investigaram-se também diferenças inter-hemisféricas relacionadas à amplitude e latência do P300 (Frizzo, Alves \& Colafêmina, 2001). Não foram encontrados resultados significativos envolvendo hemisfério e P300, o que contradiz dados publicados previamente (Alexander \& colaboradores, 1996), nos quais a amplitude do P300 é significativamente maior no hemisfério direito.
Componentes MMN e N400

Como mencionado na introdução, apenas um estudo com o intuito de investigar o componente MMN foi encontrado na literatura nacional, tendo o mesmo examinado a contribuição desse componente para a avaliação de esclerose múltipla. Os achados desse estudo demonstraram que a ausência do efeito MMN se correlaciona com a presença de comprometimento cognitivo devido a esclerose múltipla (Santos \& colaboradores, 2006).

O componente N400, tipicamente encontrado em estudos relacionados a linguagem, foi investigado em três estudos desenvolvidos no Brasil. Um desses estudos teve como objetivo examinar a abordagem estatística usualmente empregada na análise do N400 (denominada grand average). Os autores discutem problemas inerentes à utilização dessa abordagem para o estudo do N400, isto é, limitações desta abordagem para captar variações entre indivíduos na manifestação desse efeito. É sugerido, por fim, o emprego de uma ferramenta metodológica que estima automaticamente a latência e a amplitude do N400 e, consequentemente, oportuniza que essas variações entre os participantes sejam examinadas estatisticamente mediante testes não-paramétricos (Cagy, Infantosi, França \& Lemle, 2006). Outro estudo investigou a influência de variáveis sintáticas na eliciação do N400 (França, Lemle, Cagy, Constant \& Infantosi, 2004), ou seja, comparou a ativação do N400 eliciado por sentenças congruentes e incongruentes que variavam no que se refere à construção sintática. Esse estudo demonstrou importantes mudanças nos efeitos PRE de acordo com essas variações sintáticas, o que, de acordo com os autores, proporciona evidências para um modelo teórico no qual o processamento da linguagem é o resultado de operações específicas geradas pela tarefa e envolve subsistemas neurobiológicos distintos. Um terceiro estudo, conduzido por Fonseca, Tedrus e Gilbert (2006), investigou esse componente por meio de uma tarefa de decisão lexical (palavra versus pseudopalavra) realizada por crianças. Foi demonstrado que as pseudopalavras eliciavam um efeito N400 diferenciado em relação às palavras, sugerindo ainda que esse efeito iniciava mais rapidamente em crianças mais velhas em relação a crianças mais jovens.

\section{Análise crítica}

A partir dos resultados da presente busca bibliográfica, torna-se evidente que a investigação do efeito P300 é o tipo de estudo com extração de PREs até então mais frequentemente realizado nas universidades brasileiras. Consequentemente, esses estudos abordam diversas questões de pesquisa, 
incluindo a normatização desse efeito em uma amostra de indivíduos saudáveis (Veiga \& colaboradores, 2004), investigações sobre desenvolvimento e envelhecimento (Viana-Wackermann \& colaboradores, 2007; Matas \& cols., 2006), investigações sobre o efeito de determinadas substâncias sobre esse componente (Puga \& colaboradores, 2005), influência de determinados diagnósticos clínicos sobre a amplitude e latência desse componente (Reis \& Iório, 2007), aspectos metodológicos relativos à sua análise (Junqueira \& Colafêmina, 2002) e questões quanto à diferença inter-hemisférica na manifestação desse efeito (Frizzo, Alves \& Colafêmina, 2001). Os resultados proporcionados por esses estudos são de grande relevância no que se refere aos temas abordados pelos mesmos, e reafirmam a utilidade da extração e análise de P300 para a investigação de correlatos eletrofisiológicos de variados fenômenos clínicos e psicológicos (Polich, 2007).

No que se refere a estudos nacionais buscando a extração do componente MMN, somente um foi encontrado (Santos \& colaboradores, 2006). Este demonstrou uma correlação entre a ausência desse efeito com a presença de comprometimento cognitivo resultante de esclerose múltipla. Quanto aos três estudos encontrados na literatura nacional que investigaram o componente $\mathrm{N} 400$, um investigou variáveis metodológicas referentes à análise desse componente (Cagy \& cols., 2006); outro, variáveis sintáticas através da extração do componente (França, Lemle, Cagy, Constant \& Infantosi, 2004) e outro examinou a manifestação desse componente em crianças por meio de uma tarefa de decisão lexical (Fonseca, Tedrus \& Gilbert, 2006). É importante destacar que os estudos nacionais que investigaram MMN e N400 adicionam dados importantes para a literatura em PRE e processamento da linguagem. Um exemplo importante disso consiste no desenvolvimento de uma abordagem estatística alternativa e potencialmente mais eficiente para a análise do efeito N400 (Cagy \& cols., 2006). Outro exemplo é a constatação de que esse efeito, quando eliciado pela discriminação de palavras e pseudopalavras, é mais precoce em crianças com mais idade em comparação com crianças mais jovens, o que sugere que crianças em etapas mais iniciais do desenvolvimento necessitam mais tempo em sua tentativa de ativar o sentido das palavras enquanto tentam discriminá-las (Fonseca \& cols., 2006).

A extração e análise dos componentes P300, MMN e N400 são procedimentos amplamente utilizados em pesquisa durante as últimas décadas (Sutton, Braren, Zubin \& John, 1965; Kutas \& Hillyard, 1980; Coles \& Rugg, 1995). Por isso, oferecem algumas vantagens importantes para os pesquisadores, como a frequente replicabilidade dos seus efeitos, uma vez que o protocolo experimental adequado seja utilizado (Picton \& colaboradores, 2000), e o respaldo da literatura referente aos processos psicológicos subjacentes a esses componentes (Polich, 2007). Entretanto, abordagens alternativas e metodologias mais sofisticadas podem ser mais proveitosas quando se tem como objetivo investigar processos psicológicos básicos e seus correspondentes mecanismos neurais (Rugg, 2005). Protocolos experimentais provindos da pesquisa em psicologia cognitiva podem ser utilizados com o objetivo de se investigarem os correlatos neurobiológicos de fenômenos de interesse (por exemplo: atenção dividida) (Picton \& colaboradores, 2000). Essa abordagem proporciona, assim, uma variável dependente complementar ao pesquisador interessado em cognição, pois além das respostas comportamentais dos participantes, proporcionadas pela tarefa experimental, as ativações eletrofisiológicas geradas pelo cérebro oferecem informações que podem evidenciar, por exemplo, o emprego de diferentes processos cognitivos ante diferentes condições experimentais (Henson, 2005).

Sendo assim, abordagens de caráter mais exploratório, nas quais se examinam efeitos eletrofisiológicos resultantes de tarefas psicológicas experimentais, podem proporcionar dados com maior ineditismo e relevância para o desenvolvimento teórico contemporâneo da psicologia cognitiva e da neurociência (Rugg, 1997), o que pode ser extremamente proveitoso para o desenvolvimento dessas ciências no Brasil. Um exemplo das contribuições deste tipo de abordagem se refere aos efeitos eletrofisiológicos eliciados durante o processo de recuperação de memórias (Jaeger \& Parente, 2008). Isto é, a utilização de PREs tem sido fundamental para a compreensão e distinção de processos cognitivos que ocorrem enquanto indivíduos recuperam memórias episódicas recentes (Rugg, 2005), como, por exemplo, processos que precedem a recuperação do item memorizado (Johnson, Minton \& Rugg, 2008) e processos relacionados à tomada de decisão referente ao item relembrado (Rugg \& Curran, 2007). Outros exemplos de questões teóricas relevantes para a psicologia cognitiva e para a neurociência humana investigadas através de PRE são a pré-ativação (Pickering \& Schweinberger, 2003), interações envolvendo memória e emoções (Jaeger, Johnson, Corona \& Rugg, 2009) e processos perceptuais (Handy, Soltani \& Mangun, 2001).

Outro aspecto referente às possibilidades metodológicas oferecidas pelos PREs que proporciona 
informações relevantes referentes aos mecanismos neurais subjacentes a variados processos cognitivos merece ser discutido neste ponto. Esse aspecto metodológico é denominado análise topográfica (Wilding, 2000). Para esse tipo de análise, uma quantidade elevada de eletrodos (em geral, acima de 30) é utilizada, com o intuito de se examinarem os efeitos eletrofisiológicos manifestos em diferentes regiões do escalpo (Rugg, 1997). Embora na atualidade a extração de PREs não possa demonstrar com segurança a localização cerebral dos efeitos captados sobre o escalpo, problema que é frequentemente denominado "problema inverso" (Snyder, 1991), a técnica de análise topográfica pode proporcionar evidências de que dois processos cognitivos diferentes (por exemplo, memória implícita e explícita) são sustentados por regiões cerebrais distintas por apresentarem padrões dissociados de ativação sobre o escalpo. Em outras palavras, a análise topográfica não gera informações precisas referentes quanto às regiões cerebrais geradoras dos componentes, porém pode demonstrar com segurança que dois efeitos se originam de diferentes regiões do cérebro (Wilding, 2000). É importante ressaltar ainda que esse tipo de análise tem sido amplamente utilizado em importantes estudos com extração de PRE e, junto com a análise da latência, proporciona que se estabeleçam dissociações entre determinados processos cognitivos em estudo, levando em conta a distribuição espacial das ativações sobre o escalpo (Rugg \& Curran, 2007).

Apesar da importância da análise topográfica na literatura em PREs (Rugg \& Curran, 2007; Wilding, 2000), não foram encontrados na literatura nacional estudos que utilizassem esse tipo de análise. Um dos motivos para isso pode ser a utilização de um número menor de eletrodos nos estudos nacionais. Porém, mesmo quando uma quantidade suficiente de eletrodos foi utilizada, como no estudo desenvolvido por França e colaboradores (2004), esse tipo de análise não foi empregada.

É importante ressaltar que os estudos nacionais encontrados na literatura são altamente relevantes para a construção teórica em PRE. Todos possuem objetivos bem-definidos, utilizam a metodologia adequada para alcançar esses objetivos e apresentam uma análise de dados que aponta para resultados importantes. Estudos futuros, entretanto, podem ser ainda mais proveitosos para algumas áreas do conhecimento, como a psicologia cognitiva e a intersecção desta com a neurociência (Rugg, 1997), se questões teóricas centrais às mesmas forem mais diretamente abordadas, e análises alternativas e mais sofisticadas dos PREs forem utilizadas.
Por fim, é possível que relatos de pesquisa relevantes não tenham sido incluídos na presente revisão por não estarem indexados aos sites de busca citados na introdução. Por isso, pesquisas futuras podem ser importantes para investigar dados provenientes de fontes bibliográficas alternativas, assim como temas próximos não incluídos aqui, como a utilização de potenciais oscilatórios de EEG e EEG quantitativo.

\section{Considerações finais}

Os estudos nacionais que utilizam a extração e análise de PREs apresentam dados relevantes para diversas áreas do conhecimento, como, por exemplo, psicolinguística (França e colaboradores, 2004), desenvolvimento humano (Matas \& cols., 2006) e neuroquímica (Puga \& colaboradores, 2005). No que tange a abordagem metodológica desses estudos, os mesmos tiveram como variável dependente a extração de componentes específicos (P300, MMN ou N400) previamente descritos na literatura (Coles \& Rugg, 1995). O desenvolvimento das ciências cognitivas no Brasil pode se beneficiar ainda mais da utilização de PREs, se, em conjunto com a extração de componentes específicos, abordagens metodológicas alternativas e mais sofisticadas forem empregadas (Rugg, 1997). Como mencionado anteriormente, um exemplo disso consiste na análise dos PREs extraídos mediante utilização de paradigmas experimentais provindos da psicologia cognitiva, como a complementação de tríades (Badgaiyan \& Posner, 1997). Outro exemplo consiste na implementação de análises em que a distribuição topográfica dos efeitos é observada (Wilding, 2000), tendo esse tipo de análise sido crucial para a compreensão atual dos correlatos neurais de determinados processos psicológicos, como, por exemplo, memória de reconhecimento (Rugg \& Curran, 2007).

Em síntese, é importante destacar que o uso de PREs é apenas uma dentre diferentes abordagens de pesquisa disponíveis para o estudo da cognição humana. $\mathrm{O}$ desenvolvimento da psicologia cognitiva no Brasil, por exemplo, tem se beneficiado primordialmente de estudos em que dados comportamentais, como desempenho e tempo de reação, são computados e analisados (Stein \& Pergher, 2001). Do mesmo modo, estudos nacionais que analisam aspectos cognitivos de pacientes neurológicos e indivíduos idosos por testagem neuropsicológica têm gerado conhecimentos fundamentais para $\mathrm{O}$ desenvolvimento teórico da neuropsicologia (Wagner \& Parente, 2009). O emprego de PREs, dessa forma, apresenta-se como uma alternativa que complementa 
essas abordagens e, por permitir que a atividade cerebral humana seja monitorada em tempo real durante tarefas psicológicas, proporciona informações extremamente valiosas para o pesquisador interessado em cognição.

\section{Referências}

Alexander, J. E., Bauer, L. O., Kuperman, S., Morzorati, S., O'Connor, S. J., Rohrbaugh, J., Porjesz, B., Begleiter, H. \& Polich, J. (1996). Hemispheric differences for P300 amplitudes from an auditory oddball task. International Journal of Psychophysiology, 21(2-3), 189-196.

Alvarenga, K. F., Duarte, J. L., Silva, D. P. C., Agostinho-Pesse, R. S., Negrato, C. A. \& Costa, O. A. (2005). Potencial cognitivo P300 em indivíduos com diabetes mellitus. Revista Brasileira de Otorrinolaringologia, 71(2), 202-207.

Aquino, A. M. C. M., Bardão, R., Colafêmina, J. F., Gonçalvez, A. S. \& Casagrande-Souza, V. M. R. (2000). O potencial endógeno nos distúrbios de atenção e memória auditivas. Revista Brasileira de Otorrinolaringologia, 66(3), 225-230.

Badgaiyan, R. D. \& Posner, M. I. (1997). Time course of cortical activations in implicit and explicit recall. The Journal of Neuroscience, 17(12), 49044913.

Bailey, D. L., Townsend, D. W., Valk, P. E. \& Maisey, M. N. (2005). Positron emission tomography: basic sciences. Londres: Springer-Verlag.

Bressler, S. L. (2002). Event-related potentials. Em M. A. Arbib (Org.). The handbook of brain theory and neural networks (pp. 412-415). Cambridge: MIT Press.

Cagy, M., Infantosi, A. F. C., França, A. I. \& Lemle, M. (2006). Statistical analysis of event-related potentials elicited by verb-complement merge in brazilian portuguese. Brazilian Journal of Medical and Biological Research, 39(11), 1465-1474.

Colafêmina, J. F., Fellipe, A. C. N., Junqueira, C. A. O. \& Frizzo, A. C. (2000). Potenciais evocados auditivos de longa latência (P300) em adultos jovens saudáveis: um estudo normativo. Revista Brasileira de Otorrinolaringologia, 66(2), 144-148.

Coles, M. G. H. \& Rugg, M. D. (1995). Event-related brain potentials: an introduction. Em M.D. Rugg \& M.G.H. Coles (Orgs.). Electrophysiology of mind: event-related brain potentials and cognition (pp. 1-26). Oxford: Oxford University Press.
Deslandes, A. C., Veiga, H., Cagy, M., Piedade, R., Pompeu, F. \& Ribeiro, P. (2005). Effects of caffeine on the eletrophisiological, cognitive and motor responses of the central nervous system. Brazilian Journal of Medical and Biological Research, 38(7), 1077-1086.

Donchin, E. \& Coles, M. G. H. (1988). Is the P300 component a manifestation of context updating? Behavioural Brain Sciences, 11(1), 357-374.

Duarte, J. L., Alvarenga, K. F. \& Costa, O. A. (2004) Potencial cognitivo P300 realizado em campo livre: aplicabilidade do teste. Revista Brasileira de Otorrinolaringologia, 70(6), 780-785.

Fonseca, L. C., Tedrus, G. M. A. S. \& Gilbert, M. A. P. (2006). Event related potentials during the visual discrimination of words and pseudowords by children. Arquivos de Neuropsiquiatria, 64(3-A), 553558 .

França, A. I., Lemle, M., Cagy, M., Constant, P. \& Infantosi, A. F. C. (2004). Discriminating among different types of verb-complement merge in brazilian portuguese: an ERP study of morphosynthatic sub-processes. Journal of Neurolinguistics, 17(6), 425-437.

Franco, G. (2001). O potencial evocado cognitivo em adultos normais. Arquivos de Neuropsiquiatria, 59(2A), $198-200$.

Frizzo, A. C. F., Alves, R. P. C. \& Colafêmina, J. F. (2001). Potenciais evocados auditivos de longa latência: um estudo comparativo entre hemisférios cerebrais. Revista Brasileira de Otorrinolaringologia, 67(5), 618-625.

Frodl-Bauch, T., Bottlender, R. \& Hegerl, U. (1999). Neurochemical substrates and neuroanatomical generators of the event-related potentials P300. Neuropsychobiology, 40(2), 86-94.

Handy, T. C. (2004). Event-related potentials: a methods handbook. Nova Iorque: The Bradford Books.

Handy, T. C., Soltani, M. \& Mangun, G. R. (2001). Perceptual load and visuocortical processing: event-related potentials reveal sensory-level selection. Psychological Science, 12(3), 213-218.

Hegerl, U. (2004). Event-related potentials in psychiatry. Em E. Niedermeyer \& F.H. Lopes da Silva (Orgs.). Electroencephalography: basic principles, clinical applications, and related fields (pp. 621-636). Nova Iorque: Lippincott Willians \& Wilkins. 
Henson, R. (2005). What can functional neuroimaging tell the experimental psychologist? The Quarterly Journal of Experimental Psychology, 58 A(2), 193-233.

Huettel, S. A., Song, A. W. \& McCarthy, G. (2004). Functional magnetic resonance imaging. Sunderland: Sinauer Associates.

Jaeger, A. \& Parente, M. A. M. P. (2008). Event-related potentials and the study of memory retrieval: a critical review. Dementia \& Neuropsychologia, 2(4), 248-255.

Jaeger, A., Johnson, J. D., Corona, M. \& Rugg, M. D. (2009). ERP correlates of the incidental retrieval of emotional information: effects of study-test delay. Brain Research, 1269(1), 105-113.

Johnson, J. D., Minton, B. R. \& Rugg, M. D. (2008). Content dependence of the electrophysiological correlates of recollection. Neuroimage, 39(1), 406416.

Junqueira, C. A. O. \& Colafêmina, J. F. (2002). Investigação $\mathrm{da}$ estabilidade inter $\mathrm{e}$ intra examinador na identificação do P300 auditivo: análise de erros. Revista Brasileira de Otorrinolaringologia, 68(4), 468-478.

Kutas, M. \& Hillyard, S. A. (1980). Reading senseless sentences: brain potentials reflect semantic incongruity. Science, 207(4427), 203-205.

Kutas, M. \& Dale, A. (1997). Electrical and magnetic readings of mental functions. Em M. D. Rugg (Org.). Cognitive neuroscience (pp. 197-242). Cambridge: The MIT press.

Lopes da Silva, F. (2004). Dynamics of EEGs as signals of neuronal populations: models and theoretical considerations. Em E. Niedermeyer \& F. Lopes da Silva (Orgs.). Electroencephalography: basic principles, clinical applications, and related fields (pp. 76-92). (5 ed.). Nova Iorque: Lippincott Willians \& Wilkins.

Matas, C. G., Juan, K. R. \& Nakano, R. A. (2006). Potenciais evocados auditivos de média e longa latência em adultos com AIDS. Pró-fono Revista de Atualização Cientifica, 18(2), 171-176.

Matas, C. G., Santos Filha, V. A. V., Okada, M. M. C. P. \& Resque, J. R. (2006). Potenciais evocados auditivos em indivíduos acima de 50 anos de idade. Pró-fono Revista de Atualização Científica, 18(3), 277-284.

Montenegro, M., Veiga, H., Deslandes, A., Cagy, M., McDowell, K., Pompeu, F., Piedade, R. \& Ribeiro, P. (2005). Neuromodulatory effects of caffeine and bromazepan on visual event-related potentials (P300): a comparative study. Arquivos de Neuropsiquiatria, 63(2-B), 410-415.

Niedermeyer, E. \& Lopes da Silva, F. (Orgs.). (2004). Electroencephalography: basic principles, clinical applications, and related fields. (5 $5^{\text {th }}$ ed.). Nova Iorque: Lippincott Willians \& Wilkins.

Nunez, P. L. \& Srinivasan, R. (2006). Electric fields of the brain: the neurophysics of EEG. Oxford: Oxford University Press.

Pickering, E. C. \& Schweinberger, S. R. (2003). N200, $\mathrm{N} 250$ r, and N400 event-related brain potentials reveal three loci of repetition priming for familiar names. Journal of Experimental Psychology: Learning, Memory and Cognition, 29(6), 1298-1311.

Picton, T. W. (1992). The P300 wave of the human event-related potential. Journal of Clinical Neurophysiology, 9(4), 456-479.

Picton, T. W., Bentin, S., Berg, P., Donchin, E., Hillyard, S. A., Johnson, R., Miller, G. A., Ritter, W., Ruchkin, D. S., Rugg, M. D. \& Taylor, M. J. (2000). Guidelines for using human event-related potentials to study cognition: recording standards and publication criteria. Psychophysiology, 37(2), 127-152.

Polich, J. (2007). Updating P300: An integrative theory of P3a and P3b. Clinical Neurophysiology, 118(10), 2128-2148.

Pompéia, S., Bueno, O. F., Lucchesi, L. M., Manzano, G. M., Galduróz, J. C. \& Tufik, S. (2000). A double-dissociation of behavioural and eventrelated potential effects of two benzodiazepines with similar potencies. Journal of Psychopharmacology, 14(3), 288-298.

Puga, F., Veiga, H., Cagy, M., McDowell, K., Piedade, R. \& Ribeiro, P. (2005). Analysis of the influence of bromazepan on cognitive performance through the visual evoked potentials (P300). Arquivos de Neuropsiquiatria, 63(2-A), 228-234.

Reis, A. C. M. B. \& Iório, M. C. M. (2007). P300 em sujeitos com perda auditiva. Pró-fono Revista de Atualização Científica, 19(1), 113-122.

Rugg, M. D. (1997). Cognitive neuroscience. Cambridge: The MIT Press.

Rugg, M. D. (2005). Retrieval processing in human memory: electrophysiological and fMRI evidence. Em M. S. Gazzaniga (Org.). The cognitive neurosciences III (pp. 789-802). Nova Iorque: The MIT press. 
Rugg, M. D. \& Curran, T. (2007). Event-related potentials and recognition memory. Trends in Cognitive Sciences, 11(6), 251-257.

Santos, M. A. R., Munhoz, M. S. L., Peixoto, M. A. L., Haase, V. G., Rodrigues, J. L. \& Resende, L. M. (2006). Contribuição do Mismatch Negativity na avaliação cognitiva de indivíduos portadores de esclerose múltipla. Revista Brasileira de Otorrinolaringologia, 72(6), 800-807.

Shochat, E., Scheuer, C. I. \& Andrade, E. R. (2002). ABR and auditory P300 findings in children with ADHD. Arquivos de Neuropsiquiatria, 60(3-B), 742747.

Snyder, A. Z. (1991). Dipole source localization in the study of EP generators: a critique. Electroencephalography Clinical Neurophysiology, 80(4), 321-325.

Stein, L. M. \& Pergher, G. K. (2001). Criando falsas memórias em adultos por meio de palavras associadas. Psicologia: Reflexão e Crítica, 14(2), 353366.

Sutton, S., Braren, M., Zubin, J. \& John, E. R. (1965). Evoked-potentials correlates of stimulus uncertainty. Science, 150(700), 1187-1188.

Thaker, G. K. (2008). Neurophysiological endophenotypes across bipolar and schizofrenia psychosis. Schizofrenia Bulletin, 34(4), 790-773.

Turner, R., Howseman, A., Rees, G. E., Josephs, O. \& Friston, K. (1998). Functional magnetic resonance imaging of the human brain: data acquisition and analysis. Experimental Brain Research, 123(1-2), 512.
Veiga, H., Deslandes, A., Cagy, M., McDowell, K., Pompeu, F., Piedade, R. \& Ribeiro, P. (2004). Visual event-related potentials (P300): a normative study. Arquivos de Neuropsiquiatria, 62(3A), 575-581.

Viana-Wackermann, P. C., Furtado, E. F., Esser, G., Schmidt, M. H. \& Laucht, M. (2007). Lower P300 amplitude in eight year old offspring of alcoholic fathers with a delinquency history. European Archives of Psychiatry and Clinical Neuroscience, 257(4), 211-216.

Visioli-Mello, J. F. \& Rotta, N. T. (2000) Avaliação pelo P300 de crianças com e sem epilepsia e rendimento escolar. Arquivos de Neuropsiquiatria, 58(2-B), 476-484.

Wagner, P. G. Parente, M. A. M. P. (2009). O desempenho de idosos quanto a tomada de decisão em duas variações do Iowa Gambling Test. Psicologia: Teoria e Pesquisa, 25(3), 425-433.

Wilding, E. L. (2000). The practice of rescaling scalprecorded event-related potentials. Biological Psychology, 72(3), 325-332.

Recebido em julho de 2009

Reformulado em fevereiro de 2010 Aprovado em abril de 2010 
Sobre os autores:

Antonio Jaeger possui graduação em Psicologia pela Pontifícia Universidade Católica do Rio Grande do Sul (PUCRS) (2000), mestrado em Psicologia pela PUCRS (2003) e doutorado em Psicologia pela Universidade Federal do Rio Grande do Sul (UFRGS), com período sanduíche na Universidade da California, Irvine (UCI) (2007). Trabalha sob a perspectiva teórica da Psicologia Cognitiva, atuando principalmente nos seguintes temas: memória, neuroimagem e eletrofisiologia. Atualmente faz Pós-Doutorado na Washington University in St. Louis, nos Estados Unidos, onde emprega Ressonância Magnética Funcional no estudo da memória e tomada de decisões.

Maria Alice de Mattos Pimenta Parente possui graduação em Fonoaudiologia pela Pontifícia Universidade Católica de São Paulo (1972) e doutorado em Psicologia pela Universidade de São Paulo (1990). Realizou estágios de pós-doutoramento em Montreal, Canadá (1990-1992); Universidade de Toulouse, França (20012002) e Universidade de Pequim, China (2005). Atualmente é professora aposentada, colaboradora do PPG em Psicologia da Universidade Federal do Rio Grande do Sul. Tem experiência na área de Psicologia, com ênfase em Neuropsicologia, atuando principalmente nos seguintes temas: neuropsicologia, memória, compreensão textual, envelhecimento e neuropsicologia cognitiva. 